“ (C) 2016 IEEE. Personal use of this material is permitted. Permission from IEEE must be obtained for all other uses, in any current or future media, including

reprinting/republishing this material for advertising or promotional purposes, creating new collective works, for resale or redistribution to servers or lists, or reuse of any copyrighted component of this work in other works." 


\title{
Parallel distinguishability of quantum operations
}

\author{
Runyao Duan*†, Cheng Guo*, Chi-Kwong Li ${ }^{\ddagger}$, and Yinan $\mathrm{Li}^{*}$ \\ ${ }^{*}$ Center for Quantum Computation and Intelligent Systems \\ Faculty of Engineering and Information Technology, \\ University of Technology Sydney, New South Wales, Australia 2007 \\ ${ }^{\dagger}$ UTS-AMSS Joint Research Laboratory for Quantum Computation and Quantum Information Processing \\ Academy of Mathematics and Systems Science \\ Chinese Academy of Sciences, Beijing 100190, China \\ $\ddagger$ Department of Mathematics \\ College of William and Mary, Virginia, 23187-8795, USA
}

\begin{abstract}
We focus on distinguishing two quantum operations using parallel scheme. We prove that the parallel distinguishability only depends on an operator subspace that is generated by their Choi-Kraus operators. Moreover, when studying the parallel distinguishability, we can simply focus on the properties of operator subspace rather than quantum operations since any such operator subspace can be generated by the Choi-Kraus operators of two quantum operations. We provide a sufficient and necessary condition for the parallel distinguishability of two classes of operator subspaces, the one-dimensional operator spaces and the Hermitian operator space. Specifically, we give an optimal solution for one-dimensional case. However, we construct a non-trivial example to show this condition is only necessary in general.
\end{abstract}

\section{INTRODUCTION}

The distinguishability of quantum operations (or intuitively quantum devices) has received great interest in recent years. Compared with the discrimination of quantum states, which is completely characterized by their orthogonality, the distinguishability of quantum operations is more complicated but interesting. In fact, we can choose arbitrary input states as well as arbitrary schemes when distinguishing them. It has been shown that the use of entanglement can significantly improve the discrimination efficiency [1], [2], [7], [8], [10]. Meanwhile, it has also been shown that by using a sequential scheme, entanglement is not always necessary when distinguishing unitary operations [3]. Thus there is an interesting trade-off between the spatial resources (entanglement or circuits) and the temporal resources (running steps or discriminating time) when distinguishing quantum operations. More precisely, we consider two basic strategies, the adaptive strategy and the non-adaptive strategy [5]. Adaptive strategies allow us to reuse the outputs of previous uses of the quantum operation when preparing the input to subsequent uses; While non-adaptive strategies require that the inputs to all uses of the given operation are chosen before any of them is applied with possible auxiliary systems.

It is worth noting that a sufficient and necessary condition for the perfect distinguishability of quantum oper-

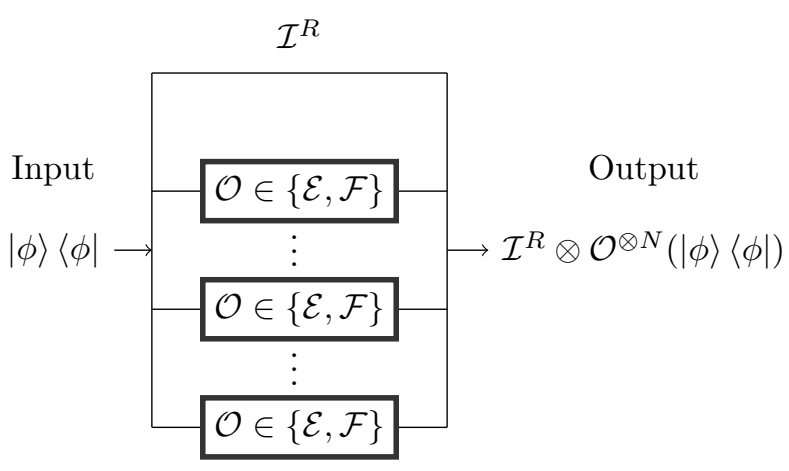

Figure 1. A parallel scheme to distinguish an unknown quantum operation $\mathcal{O} \in\{\mathcal{E}, \mathcal{F}\}$ with $N$ uses, where $\mathcal{I}^{R}$ represents the identity operator on the auxiliary system $R$.

ations has been obtained when general adaptive discrimination strategy is used [4]. However, in practice available resources for discrimination could be very limited and it is not always possible to use adaptive strategies. For instance, consider the scenario that Alice and Bob are separated by a long distance and share an unknown quantum channel which needs to be identified. When an adaptive protocol is applied, Bob needs to send the intermediate outputs back to Alice for preparing the next input, which requires more resources and infeasible. Clearly nonadaptive strategy would be more suitable in this situation.

In this paper we focus on non-adaptive strategy, or the parallel scheme, which only allows to use the unknown operation in parallel. An auxiliary system can also be used if needed, as shown in Figure I. More precisely, we consider distinguishing two quantum operations $\mathcal{E}$ and $\mathcal{F}$ with respective Choi-Kraus operators $\left\{E_{j}: j=1, \ldots, n_{0}\right\}$ and $\left\{F_{k}: k=1, \ldots, n_{1}\right\}$ in parallel. We first show that parallel distinguishability is equivalent to the existence of an integer $N$ such that there is a density operator 
$\rho \in\left(S_{\mathcal{E}, \mathcal{F}}^{\otimes N}\right)^{\perp}$, where

$$
S_{\mathcal{E}, \mathcal{F}}=\operatorname{span}\left\{E_{j}^{\dagger} F_{k}: j=1, \ldots, n_{0}, k=1, \ldots, n_{1}\right\} .
$$

We will also show that $S_{\mathcal{E}, \mathcal{F}}$ can be chosen as arbitrary operator subspace. Moreover, we will propose a sufficient and necessary condition for two classes of operator subspaces, Namely the one-dimensional operator space and the Hermitian operator space. Specifically, in both of these cases we can always obtain the optimal number of times which we need to tensor. However, this condition is only necessary for general cases.

\section{THE CHARACTERIZATION OF PARALLEL DISTINGUISHABILITY}

Consider a $d$-dimensional Hilbert space $\mathcal{H}_{d}$. The set of all linear operators on $\mathcal{H}_{d}$ is denoted by $\mathcal{B}\left(\mathcal{H}_{d}\right)$. A general quantum state $\rho$ on $\mathcal{H}_{d}$ is a density operator in $\mathcal{B}\left(\mathcal{H}_{d}\right)$ which is positive with trace unity. Moreover, a pure state $|\psi\rangle$ is a unit vector in $\mathcal{H}_{d}$ and the set of all density operators in $B\left(\mathcal{H}_{d}\right)$ is denoted by $\mathcal{D}\left(\mathcal{H}_{d}\right)$. Let $\rho$ have the spectral decomposition $\rho=\sum_{k=1}^{d} \lambda_{k}\left|\psi_{k}\right\rangle\left\langle\psi_{k}\right|$. The support of $\rho$ is defined by $\operatorname{supp}(\rho)=\operatorname{span}\left\{\left|\psi_{k}\right\rangle: \lambda_{k}>0\right\}$. Moreover, the Hilbert-Schmidt inner product for $A, B \in \mathcal{B}\left(\mathcal{H}_{d}\right)$ is given by $\operatorname{Tr}\left(A^{\dagger} B\right)$.

A quantum operation $\mathcal{E}$ from $\mathcal{B}\left(\mathcal{H}_{d}\right)$ to $\mathcal{B}\left(\mathcal{H}_{d^{\prime}}\right)$ is a completely positive and trace-preserving (CPTP) map with the form $\mathcal{E}(\rho)=\sum_{i=1}^{n} E_{i} \rho E_{i}^{\dagger}$, where $\left\{E_{i}: i=\right.$ $1, \ldots, n\}$ are the Choi-Kraus operators of $\mathcal{E}$ satisfying $\sum_{i=1}^{n} E_{i}^{\dagger} E_{i}=I_{d}$. An isometry operation is a quantum operation with only one Choi-Kraus operator $U$ such that $U^{\dagger} U=I_{d}$. $U$ is a unitary operation when $d=d^{\prime}$.

We first consider the condition under which two quantum operations $\mathcal{E}$ and $\mathcal{F}$ from $\mathcal{B}\left(\mathcal{H}_{d}\right)$ to $\mathcal{B}\left(\mathcal{H}_{d^{\prime}}\right)$ can be distinguished with one single use. In other words, we want to find a normalized pure input (possibly entangled) state $|\phi\rangle^{R Q}$ such that $\left(\mathcal{I}_{d}^{R} \otimes \mathcal{E}^{Q}\right)\left(|\phi\rangle\left\langle\left.\phi\right|^{R Q}\right)\right.$ and $\left(\mathcal{I}_{d}^{R} \otimes \mathcal{F}^{Q}\right)\left(|\phi\rangle\left\langle\left.\phi\right|^{R Q}\right)\right.$ are orthogonal, where $\mathcal{H}_{d}^{R}$ denote the auxiliary system and $\mathcal{H}_{d}^{Q}$ is the principle system under consideration. Moreover, we assume $|\phi\rangle^{R Q}=\left(I^{R} \otimes X^{Q}\right)|\Psi\rangle$ where $X$ satisfies $\operatorname{Tr}\left(X^{\dagger} X\right)=1$ and $|\Psi\rangle=\sum_{i=0}^{d-1}\left|i^{R}\right\rangle\left|i^{Q}\right\rangle$ which is the (un-normalized) maximally entangled state between $\mathcal{H}_{d}^{R}$ and $\mathcal{H}_{d}^{Q}$. By substituting $\mathcal{E}$ and $\mathcal{F}$ with their Choi-Kraus operators $\left\{E_{j}: j=1 \ldots n_{0}\right\}$ and $\left\{F_{k}: k=1 \ldots n_{1}\right\}$ and using the Hilbert-Schmidt inner product over $\mathcal{B}\left(\mathcal{H}_{d} \otimes \mathcal{H}_{d}^{\prime}\right)$, we obtain

$$
\sum_{j, k} \operatorname{Tr}\left(E_{j}^{\dagger} F_{k} X X^{\dagger}\right) \operatorname{Tr}\left(F_{k}^{\dagger} E_{j} X X^{\dagger}\right)=0,
$$

which immediately implies that $X X^{\dagger}$ is orthogonal to $\left\{E_{j}^{\dagger} F_{k}: j=1 \ldots n_{0}, k=1 \ldots n_{1}\right\}$. Noticing $X X^{\dagger}$ is always positive and trace unity, $X X^{\dagger} \in \mathcal{D}\left(\mathcal{H}_{d}\right)$.

When multiple uses of the unknown quantum operation is considered, the calculation is similar and we obtain the following theorem
Theorem 1. Let $\mathcal{E}$ and $\mathcal{F}$ be two quantum operations from $\mathcal{B}\left(\mathcal{H}_{d}\right)$ to $\mathcal{B}\left(\mathcal{H}_{d^{\prime}}\right)$ with Choi-Kraus operators $\left\{E_{j}: j=\right.$ $\left.1 \ldots n_{0}\right\}$ and $\left\{F_{k}: k=1 \ldots n_{1}\right\}$, respectively. They can be distinguished by $N$ uses in parallel if and only if there exists a density operator $\rho \in\left(\operatorname{span}\left\{E_{j}^{\dagger} F_{k}: j=1 \ldots n_{0}, k=\right.\right.$ $\left.\left.1 \ldots n_{1}\right\}^{\otimes N}\right)^{\perp}$.

This theorem shows that the parallel distinguishability is only determined by the operator subspace $S_{\mathcal{E}, \mathcal{F}}=$ $\operatorname{span}\left\{E_{j}^{\dagger} F_{k}: j=1 \ldots n_{0}, \quad k=1 \ldots n_{1}\right\}$. Moreover, we can easily derive a necessary condition for parallel distinguishability:

Corollary 1. If $\mathcal{E}$ and $\mathcal{F}$ can be perfectly distinguished with finite uses using parallel scheme, then there is no positive definite operator in $S_{\mathcal{E}, \mathcal{F}}$.

Meanwhile, we are curious about what kind of operator subspace can be generated by two quantum operations. The following theorem shows that the operator subspace can be chosen freely.

Theorem 2. For any subspace $T \subset B\left(\mathcal{H}_{d}\right)$, there are two quantum operations $\mathcal{E}$ and $\mathcal{F}$ from $\mathcal{B}\left(\mathcal{H}_{d}\right)$ to $\mathcal{B}\left(\mathcal{H}_{d^{\prime}}\right)$ such that $T=S_{\mathcal{E}, \mathcal{F}}$.

Proof. We first assume $T$ is spanned by a finite set of operators $\left\{T_{1}, T_{2}, \ldots, T_{N}\right\}$ where $N \leq d^{2}$ is the dimension of $T$. Moreover, we assume $T_{i}^{\dagger} T_{i} \leq I_{d}$ for $i=1, \ldots, N$. We will show that for each $T_{i}$, there exists two isometries $U_{i}$ and $V_{i}$ from $\mathcal{H}_{d}$ to $\mathcal{H}_{d^{\prime}}$ where $d^{\prime} \geq 2 d$ such that $T_{i}=U_{i}^{\dagger} V_{i}$. To see this, let $T_{i}$ have the singular value decomposition $\sum_{k=1}^{n_{i}} \sigma_{i}^{k}\left|\psi_{i}^{k}\right\rangle\left\langle\phi_{i}^{k}\right|$, where $0 \leq \sigma_{i}^{k} \leq 1$.

Define $U_{i}=\sum_{k=1}^{n_{i}}\left|\alpha_{i}^{k}\right\rangle\left\langle\bar{\psi}_{i}^{k}\right|$ and $V_{i}=\sum_{k=1}^{n_{i}}\left|\beta_{i}^{k}\right\rangle\left\langle\phi_{i}^{k}\right|$. For each $i,\left\{\left|\alpha_{i}^{k}\right\rangle: 1 \leq k \leq n_{i}\right\}$ and $\left\{\left|\beta_{i}^{k}\right\rangle: 1 \leq k \leq n_{i}\right\}$ are two sets of orthonormal vectors in $\mathcal{H}_{d^{\prime}}$ to be determined. To make $U_{i}$ and $V_{i}$ satisfy $T_{i}=U_{i}^{\dagger} V_{i}$, we need:

$$
\left\langle\alpha_{i}^{j} \mid \beta_{i}^{k}\right\rangle=0 \text { and }\left\langle\alpha_{i}^{k} \mid \beta_{i}^{k}\right\rangle=\sigma_{i}^{k} \text {, for any } j, k=1, \ldots n_{i} \text {. }
$$

This can be done by choosing $n_{i}$ two-dimensional subspaces in $\mathcal{H}_{d^{\prime}}$ which are mutually orthogonal, and denote them by $K_{i}^{j}$ with $j=1, \ldots, n_{i}$. In each $K_{i}^{j}$ we choose a basis $\left\{\left|\alpha_{i}^{j}\right\rangle,\left|\beta_{i}^{j}\right\rangle\right\}$ such that $K_{i}^{j}=\operatorname{span}\left\{\left|\alpha_{i}^{j}\right\rangle,\left|\beta_{i}^{j}\right\rangle\right\}$ and $\left\langle\alpha_{i}^{j} \mid \beta_{i}^{j}\right\rangle=\sigma_{i}^{j}$. This can be done since $0 \leq \sigma_{i}^{j} \leq 1$. (In the special case of $\sigma_{i}^{j}=1, K_{i}^{j}$ is one-dimensional). Note that we can let $d^{\prime} \geq 2 d \geq \max \left\{2 n_{i}: i=1,2, \ldots, N\right\}$ so such $\left\{K_{i}^{j}\right\}$ always exist.

Now we construct two quantum operations $\mathcal{E}$ and $\mathcal{F}$ with Choi-Kraus operators $\left\{E_{j}: j=1, \ldots, N\right\}$ and $\left\{F_{k}: k=1, \ldots, N\right\}$ such that $T=S_{\mathcal{E}, \mathcal{F}}$. This can be done by choosing $E_{j}=\frac{1}{\sqrt{N}} U_{j} \otimes|j\rangle$ and $F_{k}=\frac{1}{\sqrt{N}} V_{k} \otimes|k\rangle$, where $j, k=1, \cdots, N$.

We say an operator subspace $S$ has parallel distinguishability, if there exists a finite positive integer $N$ such that there is a non-zero positive operator in the orthogonal complement of $S^{\otimes N}$. In such a case, we know that for all quantum operations $\mathcal{E}$ and $\mathcal{F}$ such that $S_{\mathcal{E}, \mathcal{F}}=S$, they can be perfect distinguished by $N$ uses in parallel. 


\section{PARALlEL DISTINGUISHABILITY OF TWO KINDS OF OPERATOR SUBSPACES}

The parallel distinguishability is more difficult than the perfect distinguishability introduced in [4], since only limited resources can be used. We want to find some efficient method to check if an operator subspace has parallel distinguishability. By Corollary 1, we know that the operator space should not have a positive definite operator. We will introduce two families of operator subspaces such that the parallel distinguishability is only determined by the existence of positive definite operator.

First, we consider the condition that $\operatorname{dim}(S)=1$. One simple case is $S=\operatorname{span}\{U\}$, where $U$ is a unitary operator. In this situation $\mathcal{E}$ and $\mathcal{F}$ can be chosen as different unitary operations. Clearly they can be distinguished in parallel [2] if and only if $U \neq I_{d}$. Let us consider $S=\operatorname{span}\{A\}$ where $A \in \mathcal{B}\left(\mathcal{H}_{d}\right)$ is not a unitary operator and not positive definite. We will use the theory of numerical range in our study. For $A \in \mathcal{B}\left(\mathcal{H}_{d}\right)$, let

$$
W(A)=\left\{\langle\psi|A| \psi\rangle:|\psi\rangle \in \mathcal{H}_{d},\langle\psi \mid \psi\rangle=1\right\} .
$$

be the numerical range of $A$, which has been researched for decades. It is known that the numerical range of an operator $A$ is always convex by the celebrated ToeplitzHausdorff Theorem; for example see [6, Chapter 1]. It is also known that the numerical range of a normal operator is just the convex hull of its eigenvalues, and $W(I \otimes A)=W(A)$. Moreover, we can define the angular numerical range:

Definition 1. For a linear operator $A \in \mathcal{B}\left(\mathcal{H}_{d}\right)$, the angular numerical range of $A$ is defined as follows:

$$
\mathcal{W}(A)=\cup_{t>0} W(t A) .
$$

By the convexity of $W(A), \mathcal{W}(A)$ can be $\mathbb{C}$, a half space with a straight line passing through 0 as the boundary, or a pointed cone with 0 as the vertex. We can define the field angle of $A$ according to these cases as follows.:

Definition 2. For a linear operator $A \in \mathcal{B}\left(\mathcal{H}_{d}\right)$, the field angle of $A$, denoted by $\Theta(A)$, is defined as follows:

1) If $\mathcal{W}(A)=\mathbb{C}, \Theta(A)=2 \pi$

2) If $\mathcal{W}(A)$ is a half space, then $\Theta(A)=\pi$;

3) If $\mathcal{W}(A)$ is a pointed cone, then $\Theta(A)$ is the angle between the two boundary rays of the cone.

Theorem 3. Consider $S=\operatorname{span}\{A\}$ where $A \in \mathcal{B}\left(\mathcal{H}_{d}\right)$. Then $S$ has parallel distinguishability if and only if for any real $t, e^{i t} A$ is not positive definite.

Proof. Suppose $W(A) \subseteq e^{i t}(0, \infty)$ for some real $t$, i.e. $e^{-i t} A$ is positive definite. Then for any positive integer $N, W\left(\left(e^{-i t} A\right)^{\otimes N}\right) \subseteq(0, \infty)$. Thus $S$ does not have parallel distinguishability.

If $0 \in W(A)$, i.e. there is $|\psi\rangle$ such that $\operatorname{Tr}(A|\psi\rangle\langle\psi|)=0$. In this situation choose $\rho=|\psi\rangle\langle\psi|$ and we are done.
If $0 \notin W(A)$ and $W(A) \nsubseteq e^{i t}(0, \infty)$ for any real $t$, then there exists a cone in $\mathbb{C}$ with vertex 0 containing $W(A)$. So, there are $\mu_{1}=r_{1} e^{i \theta_{1}}, \mu_{2}=r_{2} e^{i \theta_{2}} \in W(A)$ with $r_{1}, r_{2}>0$ and $\theta_{1}<\theta_{2}<\theta_{1}+\pi$ so that $\theta_{1} \leq \arg (\mu) \leq \theta_{2}$ for all $\mu \in W(A)$. Notice that $\Theta(A)=\theta_{2}-\theta_{1}$. We may replace $A$ by $e^{-i \frac{\theta_{1}+\theta_{2}}{2}} A$ and assume that

$$
W(A) \subseteq\left\{\mu \in \mathbb{C}:-\frac{\Theta(A)}{2} \leq \arg (\mu) \leq \frac{\Theta(A)}{2}\right\} .
$$

Let $A=H+i G$, where $H$ and $G$ are Hermitian. Then $H$ is positive definite. Suppose $U \in \mathcal{B}\left(\mathcal{H}_{d}\right)$ is unitary such that

$$
\begin{aligned}
A_{0} & =U^{\dagger} H^{-1 / 2} A H^{-1 / 2} U \\
& =U^{\dagger}\left(I_{d}+i H^{-1 / 2} G H^{-1 / 2}\right) U \\
& =\operatorname{diag}\left(1+a_{1} i, \ldots, 1+a_{d} i\right)
\end{aligned}
$$

with $a_{1} \geq \cdots \geq a_{d}$. Then $\mathcal{W}(A)=\mathcal{W}\left(A_{0}\right)$ and $a_{1}=$ $\tan \frac{\Theta(A)}{2}$ and $a_{d}=-\tan \frac{\Theta(A)}{2}$. Furthermore,

$$
\mathcal{W}\left(A^{\otimes N}\right)=\mathcal{W}\left(A_{0}^{\otimes N}\right)=\mathcal{W}\left(D^{\otimes N}\right),
$$

where $D=\operatorname{diag}\left(1+a_{1} i, 1+a_{d} i\right)$. Hence $0 \in \mathcal{W}\left(A^{\otimes N}\right)$ if and only if $0 \in \mathcal{W}\left(D^{\otimes N}\right)$. Therefore there is a non-zero positive operator in $\left\{A^{\otimes N}\right\}^{\perp}$ if and only if $N \geq \frac{\pi}{\Theta(A)}$, which is always finite. Choose $N=\left\lceil\frac{\pi}{\Theta(A)}\right\rceil$ and this will be the smallest $N$ such that there is a non-zero positive operator in $S^{\otimes N}$. Since for any positive integer $K$ smaller than $N, 0 \notin W\left(D^{\otimes K}\right)$. Thus there is no non-zero positive operator in $S^{\otimes K}$.

Moreover, we have the following:

Corollary 2. For quantum operations $\mathcal{E}$ and $\mathcal{F}$ such that $S_{\mathcal{E}, \mathcal{F}}=\operatorname{span}\{A\}$ where $A \in \mathcal{B}\left(\mathcal{H}_{d}\right)$. They can be parallel distinguished within finite use if and only if for any real $t, e^{i t} A$ is not positive definite. Moreover, the minimum number of using the unknown device is $\left\lceil\frac{\pi}{\Theta(A)}\right\rceil$.

Another family of operator subspace, the operator space spanned by a set of Hermitian operators, which has parallel distinguishability if there is no positive definite operator in this space. We have the following:

Theorem 4. For an operator subspace $S$ such that $S=S^{\dagger}$ where $S^{\dagger}=\left\{E^{\dagger}: E \in S\right\}, S$ has parallel distinguishability if and only if there is no positive definite operator in $S$.

Proof. Assume $\left\{A_{1}, \ldots, A_{N}\right\}$ is a set of Hermitian operators such that $S=\operatorname{span}\left\{A_{1}, \ldots, A_{N}\right\}$. By Farkas lemma of semi-definite programming [9], either

- There is a linear combination of $A_{1}, \ldots, A_{N}$ equal to a positive definite operator; or

- There is a non-zero positive operator $\rho$ such that $\operatorname{Tr}\left(A_{i} \rho\right)=0$ for $i=1, \ldots, N$.

The first statement is equivalent to the existence of a positive definite operator in $S$ and the second one is equivalent to $\rho \in S^{\perp}$. Thus if there is no positive definite operator in $S$, we can always find a non-zero positive operator in $S^{\perp}$. 


\section{Nonexistence of a positive Definite OPERATOR IS NOT ALWAYS SUFFICIENT}

For arbitrary operator subspace, we are curious about if the nonexistence of positive definite operator is sufficient for checking the parallel distinguishability. Unfortunately, there exists operator subspace $S$ such that there is no positive definite operator in $S$ but $S$ does not have parallel distinguishability.

Theorem 5. Let $S=\operatorname{span}\left\{A_{1}, A_{2}\right\} \in \mathcal{B}\left(\mathcal{H}_{3}\right)$ with $A_{1}=|0\rangle\langle 0|+i| 1\rangle\langle 1|$ and $A_{2}=|1\rangle\langle 1|+i| 2\rangle\langle 2|$, where $\{|0\rangle,|1\rangle,|2\rangle\}$ is an orthonormal basis of $\mathcal{H}_{3}$. Then for any positive integer $N$, there is no non-zero positive operator in the orthogonal complement of $S^{\otimes N}$.

Proof. It is easy to verify that there is no positive definite operator in $S$, we will show that for arbitrary integer $N$, there is no density operator in $\left(S^{\otimes N}\right)^{\perp}$ by mathematical induction. When $N=1$, by simple calculation there is no density operator in $S^{\perp}$. Assume for $N=k$, there is no density operator in $\left(S^{\otimes k}\right)^{\perp}$, consider tensor $k+1$ times of $S$, notice that $S^{\otimes k+1}=\operatorname{span}\left\{A_{i} \otimes M: i=1,2, M \in S^{\otimes k}\right\}$. If there exists a density operator $\rho \in\left(S^{\otimes k+1}\right)^{\perp}$, we have

$$
\operatorname{Tr}\left(\rho\left(A_{1} \otimes M\right)\right)=0, \quad \operatorname{Tr}\left(\rho\left(A_{2} \otimes M\right)\right)=0,
$$

where $M \in S^{\otimes k}$ Since $A_{1}$ and $A_{2}$ are diagonal, we may assume $\rho=|0\rangle\left\langle 0\left|\otimes \rho_{0}+\right| 1\right\rangle\left\langle 1\left|\otimes \rho_{1}+\right| 2\right\rangle\langle 2| \otimes \rho_{2}$ where $\rho_{0}$, $\rho_{1}$ and $\rho_{2}$ are positive operators and at least one of them is non-zero. By substitution we have:

$\operatorname{Tr}\left(\rho_{0} M\right)+i \operatorname{Tr}\left(\rho_{1} M\right)=0, \quad-i \operatorname{Tr}\left(\rho_{1} M\right)+\operatorname{Tr}\left(\rho_{2} M\right)=0$.

Let $\sigma=\left(\rho_{0}+\rho_{2}\right) / \operatorname{Tr}\left(\rho_{0}+\rho_{2}\right)$, then $\operatorname{Tr}(\sigma M)=0$. If $\rho_{0}+$ $\rho_{2} \neq 0$, then $\sigma$ is a non-zero positive operator in $\left(S^{\otimes k}\right)^{\perp}$, which is a contradiction. Thus $\rho_{0}=\rho_{2}=0$. But then, we can conclude that $\rho_{1}$ is non-zero and $\operatorname{Tr}\left(\rho_{1} M\right)=0$ for all $M \in S^{\otimes k}$, again a contradiction.

In general, the parallel distinguishability of an operator subspace can be checked by the following system of equations:

$$
\begin{array}{ll}
\operatorname{Tr}\left(\rho A_{j_{1}} \otimes \cdots \otimes A_{j_{N}}\right)=0 \quad & \text { for } \quad k=1, \ldots, N \\
& \operatorname{Tr}(\rho)=1, \quad \rho \geq 0,
\end{array}
$$

where $A_{j_{k}} \in S$ for $k=1, \ldots, N$ for arbitrary $N$. Notice that this can be treated as a semi-definite programming (SDP) problem. In fact, it is still not easy to check even we reduce it to a linear programming (LP) problem. We will show this by constructing a family of operator spaces with a parameter $\alpha$, let

$$
S_{\alpha}=\left\{A_{1}=|0\rangle\left\langle 0\left|+e^{i \alpha}\right| 1\right\rangle\left\langle 1\left|, A_{2}=\right| 1\right\rangle\left\langle 1\left|+e^{i \alpha}\right| 2\right\rangle\langle 2|\right\} .
$$

Let $\alpha \in[\pi / 2, \pi]$ to guarantee there is no positive definite operator in $S$ and $\{|0\rangle,|1\rangle,|2\rangle\}$ is an orthonormal basis of $\mathcal{H}_{3}$. Notice that $S_{\alpha}$ is just an operator space spanned by two $3 \times 3$ diagonal operators, which seems easy to be solved. By simple calculation we can obtain that there is a non-zero positive operator in $S_{\alpha}^{\perp}$ if and only if $\alpha=\pi$. Consider $S_{\alpha}{ }^{\otimes 2}$, the above system of linear equations has a positive solution if and only if $\alpha \in\left[\frac{3 \pi}{4}, \pi\right]$, and we can choose the diagonal entries of $\rho$ to be:

$$
(1,-\cos \alpha, \cos 2 \alpha,-\cos \alpha, 1,-\cos \alpha, \cos 2 \alpha,-\cos \alpha, 1) \text {. }
$$

However, for $N>2$, the number of equations is $2^{N}$ and the number of variables is $3^{N}$, this procedure will not be efficient. We are going to simplify the problem by using the symmetry. The basic idea to decrease the number of variables is to classify them into different types and assume the variables in each type shares the same value. More precisely, let us consider a fixed $N$ and represent the index of variables $p_{0}, \ldots, p_{3^{N}-1}$ by ternary numbers $\underbrace{0 \ldots 0}_{N}, \ldots, \underbrace{2 \ldots 2}_{N}$. We denote the ternary expansion of $k$ by $\bar{k}$ and the numbers of 0,1 and 2 in $\bar{k}$ as $\bar{k}_{0}, \bar{k}_{1}$ and $\bar{k}_{2}$. In fact, we assume all the index with same number of 0,1 and 2 are equal, i.e. $p_{k}=p_{k^{\prime}}$ if $\bar{k}_{i}={\overline{k^{\prime}}}_{i}$ for $i=0,1,2$. This can be done since $S^{\otimes N}$ is invariant under any permutation with order $N$ on its subsystems. Thus if it has a solution it must have a symmetric solution which is invariant under permutations of order $N$. Moreover, we assume:

$$
p_{k}=\frac{\bar{k}_{0} ! \bar{k}_{1} ! \bar{k}_{2} !}{N !} a_{\bar{k}_{0}, \bar{k}_{1}, \bar{k}_{2}} \text {. }
$$

By this substitution we can reduce the number of variables to $O\left(N^{2}\right)$. Moreover, we can also use this symmetry to reduce the number of equations. Considering the diagonal elements of $A_{1}^{\otimes N}$, it is easy to see the index will be all the binary number from 0 to $2^{N}-1$. Denote the binary expansion of $k$ by $\hat{k}$ and the number of 1 in $\hat{k}$ by $\hat{k}_{1}$ similarly. We can rewrite the equation $\operatorname{Tr}\left(A_{1}^{\otimes N} \rho\right)=0$ by:

$$
\sum_{j=0}^{2^{N}-1} e^{i \hat{j}_{1} \alpha} p_{j}=0
$$

Moreover, let $\mathcal{J}_{r}=\left\{j: \hat{j}_{1}=r, 0 \leq j \leq 2^{N}-1\right\}$, then $\left|\mathcal{J}_{r}\right|=\left(\begin{array}{c}N \\ r\end{array}\right)$ and $\left(\begin{array}{c}N \\ r\end{array}\right) p_{j}=a_{N-r, r, 0}$ if $j \in \mathcal{J}_{r}$. Thus we can rewrite the above equation as:

$$
\sum_{r=0}^{N} e^{i r \alpha} a_{N-r, r, 0}=0 .
$$

Moreover, by the symmetry we can ignore the place of $A_{2}$ when we substitute it with $A_{1}$. We only need to consider equations $\operatorname{Tr}\left(A_{2}^{\otimes l} \otimes A_{1}^{\otimes N-l} \rho\right)=0$ for $l=0, \ldots, N$, which reduce the number of equations to $O(N)$. Furthermore, the substitution of $A_{1}$ by $A_{2}$ will permute the index of variables in Equation 3 but remain the coefficient unchanged. More precisely, for an index $k$ with ternary expansion $\bar{k}=x_{N-1} 3^{N-1}+\cdots+x_{1} 3+x_{0}$, if we substitute the first $l A_{1}$ by $A_{2}$, the index after substitution will be $\bar{k}_{l}=\left(x_{N-1}+1\right) 3^{N-1}+\cdots+\left(x_{N-l}+1\right) 3^{N-l}+$ $x_{N-l-1} 3^{N-l-1}+\cdots+x_{0}$. Noticing that we use binary number $\hat{k}=x_{N-1} 2^{N-1}+\cdots+x_{1} 2+x_{0}$ in Equation 3 , we define the following map $\bar{f}$ to transfer a binary number $\hat{k}$ 
to a ternary number $\bar{f}(\hat{k})=x_{N-1} 3^{N-1}+\cdots+x_{1} 3+x_{0}$. Thus $\operatorname{Tr}\left(A_{2}^{\otimes l} \otimes A_{1}^{\otimes N-l} \rho\right)=0$ can be rewriten as:

$$
\sum_{j=0}^{2^{N}-1} e^{i \hat{j}_{1} \alpha} p_{\bar{f}(\hat{j})_{l}}=0
$$

Since we are going to use Equation 2 to represent $p_{\bar{f}(\hat{j})_{l}}$. We consider the elements in $\mathcal{J}_{r}$. Denote the number of 0 in the first $l$ positions of $j \in \mathcal{J}_{r}$ by $s$, where $0 \leq s \leq$ $N-r$. After the substitution we obtain $\left(\begin{array}{l}l \\ s\end{array}\right)\left(\begin{array}{c}N-l \\ N-r-s\end{array}\right)$ ternary numbers with value of

$$
\frac{(N-r-s) !(2 s+r-l) !(l-s) !}{N !} p_{N-r-s, 2 s+r-l, l-s .} .
$$

Thus we rewrite Equation 4 as:

$$
\sum_{r=0}^{N} e^{i r \alpha}\left[\sum_{s=0}^{N-r}\left(\begin{array}{c}
2 s+r-l \\
s
\end{array}\right) a_{N-r-s, 2 s+r-l, l-s}\right]=0 .
$$

After the above procedure, we can easily obtain the explicit solution for $N=3,4$ :

For $N=3$, we have the following solution:

- $a_{0,3,0}=0$

- $a_{3,0,0}=a_{0,0,3}=a_{1,2,0}=a_{0,2,1}=\sin \alpha$;

- $a_{2,1,0}=a_{0,1,2}=-\sin 2 \alpha$

- $a_{1,1,1}=-2 \sin 2 \alpha$

- $a_{1,0,2}=a_{2,0,1}=\sin 3 \alpha$.

When $\alpha \in\left[\frac{2 \pi}{3}, \frac{3 \pi}{4}\right]$, the above values are all non-negative. When $\alpha \in\left[\frac{3 \pi}{4}, \pi\right]$, we can simply use the solution for $N=$ 2 to construct the solution.

For $N=4$, we have the following solution:

- $a_{4,0,0}=a_{0,0,4}=a_{0,4,0}=1$;

- $a_{0,2,2}=a_{2,2,0}=2$;

- $a_{0,1,3}=a_{3,1,0}=a_{0,3,1}=a_{1,3,0}=-2 \cos \alpha$;

- $a_{1,1,2}=a_{2,1,1}=2 \cos 3 \alpha$;

- $a_{2,0,2}=-2 \cos 4 \alpha$

- All the rest entries are 0 .

When $\alpha \in\left[\frac{5 \pi}{8}, \frac{2 \pi}{3}\right]$, the above values are all non-negative.

Though when $N$ is large we seem can use numerical method to find the solution, it is not efficient since there are combination numbers in the equations. We are only able to test $N$ up to 18 . However, we find an interesting conjecture which may lead to a full characterization of the parallel distinguishability for this special case:

Conjecture 1. For operator space $S_{\alpha}=\{|0\rangle\langle 0|+$ $\left.e^{i \alpha}|1\rangle\langle 1|| 1\rangle,\left\langle 1\left|+e^{i \alpha}\right| 2\right\rangle\langle 2|\right\}$ with $\alpha \in\left[\frac{\pi}{2}, \pi\right]$, there is a density operator in the orthogonal complement of $\left(S_{\alpha}\right)^{\otimes N}$ if and only if $\alpha \in\left[\frac{\pi}{2}+\frac{\pi}{2 N}, \pi\right]$.

Interstingly, we have already show that this conjecture is true when $N \rightarrow \infty$. Since $S_{\frac{\pi}{2}}$ is the case in Theorem 5 , we have already show that the desired $N$ does not exist.

\section{Conclusion}

In this paper we discuss the problem of parallel distinguishability of general quantum operations. It is illustrated that the parallel distinguishability is determined by an operator subspace generated by their Choi-Kraus operators. Meanwhile, we show that the operator subspace can be chosen as an arbitrary operator subspace. We introduc the parallel distinguishability of an operator subspace and focus on characterizing operator subspaces which have this property. Furthermore, we show the parallel distinguishability of one-dimensional operator subspace and Hermitian operator space can be verified by checking if there exists a positive definite operator. However, we construct an explict example to show that this condition is not always sufficient. In general, we find a family of operator subspaces and obtaine some analytical and numerical result as well as a conjecture about the full characterization of the parallel distinguishability of these operator spaces. Unfortunately, verifying the parallel distinguishability of these operator subspaces is still difficult.

$\mathrm{RD}$ is supported in part by the Australian Research Council (ARC) under Grant DP120103776 and by the National Natural Science Foundation (NNSF) of China under Grant 61179030; furthermore in part by an ARC Future Fellowship under Grant FT120100449. CL is supported in part by the USA NSF grant DMS 1331021, the Simons Foundation Grant 351047, and the NNSF of China Grant 11571220 .

\section{REFERENCES}

[1] A. Acín, "Statistical distinguishability between unitary operations." Physical review letters, vol. 87, no. 17, p. 177901, 2001.

[2] G. M. D'Ariano, P. Lo Presti, and M. G. A. Paris, "Using Entanglement Improves the Precision of Quantum Measurements," Physical review letters, vol. 87, no. 27, p. 270404, 2001.

[3] R. Duan, Y. Feng, and M. Ying, "Entanglement is Not Necessary for Perfect Discrimination between Unitary Operations," Physical review letters, vol. 98, no. 10, p. 100503, 2007.

[4] R. Duan, Y. Feng, and M. Ying, "Perfect Distinguishability of Quantum Operations," Physical Review Letters, vol. 103, no. 21, p. 210501, 2009.

[5] A. W. Harrow, A. Hassidim, D. W. Leung, and J. Watrous, "Adaptive versus non-adaptive strategies for quantum channel discrimination," Physical Review A, vol. 81, no. 3, p. 11, 2009.

[6] R. A. Horn and C. R. Johnson, Matrix analysis. Cambridge university press, 2012.

[7] Z. Ji, Y. Feng, R. Duan, and M. Ying, "Identification and Distance Measures of Measurement Apparatus," Physical Review Letters, vol. 96, no. 20, p. 200401, 2006.

[8] M. Piani and J. Watrous, "All entangled states are useful for channel discrimination," Physical Review Letters, vol. 102, no. June, pp. 1-4, 2009.

[9] R. T. Rockafellar, Convex analysis. Princeton university press, 2015.

[10] M. F. Sacchi, "Optimal discrimination of quantum operations," Physical Review A, vol. 71, no. 6, p. 062340, 2005. 